\title{
Head-on/Near Head-on Collisions of Neutron Stars With a Realistic EOS
}

\author{
Edwin Evans ${ }^{(1)}$, A. Gopakumar ${ }^{(1,2)}$, Philip Gressman ${ }^{(1,3)}$, Sai \\ Iyer $^{(1)}$, Mark Miller ${ }^{(1,4)}$, Wai-Mo Suen ${ }^{(1,5)}$, and Hui-Min Zhang ${ }^{(1)}$ \\ ${ }^{(1)}$ McDonnell Center for the Space Sciences, Department of Physics, \\ Washington University, St. Louis, Missouri 63130 \\ ${ }^{(2)}$ Physics Department, University of Guelph, Canada \\ (3) Mathematics Department, Princeton University, Princeton, NJ 08544 \\ (4) 238-332 Jet Propulsion Laboratory, 4800 Oak Grove Drive, Pasadena, CA 91109 and \\ (5) Physics Department, Chinese University of Hong Kong, Hong Kong
}

(Dated: October 30, 2018)

\begin{abstract}
It has been conjectured that in head-on collisions of neutron stars (NSs), the merged object would not collapse promptly even if the total mass is higher than the maximum stable mass of a cold NS. In this paper, we show that the reverse is true: even if the total mass is less than the maximum stable mass, the merged object can collapse promptly. We demonstrate this for the case of NSs with a realistic equation of state (the Lattimer-Swesty EOS) in head-on and near head-on collisions. We propose a "Prompt Collapse Conjecture" for a generic NS EOS for head on and near head-on collisions.
\end{abstract}

PACS numbers: 04.25.Dm,04.30.+x,97.60.Jd,97.60.Lf

Introduction. Shapiro [1] has conjectured that two neutron stars (NSs) falling from infinity and colliding head-on would not, independent of their masses, promptly collapse to a black hole. The basic argument for the conjecture is that, until there is significant neutrino cooling, the thermal pressure generated by shock heating is always enough to support the merged object. Since neutrino cooling operates on time scales of seconds instead of the NS dynamical time scale of milliseconds, this could have significant implications on gravitational wave and neutrino emissions. The results obtained in [1] were based on a polytropic equation of state (EOS) $P=K \rho^{\Gamma}$ (with $K$ a function of the entropy), and it was suggested that the result was true for a general EOS.

We have shown in 2] that one implicit assumption used in the derivation in 1], namely that the collision can be approximated by a quasi-equilibrium process, is not valid. We carried out simulations of the head-on collision of neutron stars described by a polytropic EOS, as in the conjecture. For two $1.4 M_{\odot}$ NSs (with a polytropic index of $\Gamma=2$, initial polytropic coefficient $K$ of $1.16 \times 10^{5} \mathrm{~cm}^{5} / \mathrm{g} \mathrm{s}^{2}$ as in a typical NS model), we showed that the merged object collapsed promptly. The shock front generated in the collision does not even have time to propagate to the outer part of the merged object before it is engulfed in an apparent horizon, let alone produce enough thermal pressure to support the merged object as envisioned in the quasi-equilibrium argument of [1]. In our study in [2], the merged object had a mass well above the critical mass (the maximum mass that the EOS with the polytropic constants $\Gamma$ and $K$ given above can support). We pointed out in [2, 3] that the prompt collapse is due to the dynamical compression of the collision that is absent in the quasi-equilibrium analysis in 1, 4].

This brings up three further questions: 1 . What if the mass of the merged object is less than the critical mass? Will the dynamical compression in the collision process be strong enough to initiate a collapse? 2. What if we use a realistic EOS instead of a polytropic EOS? 3. What if we break the exact axisymmetry? In this paper we answer these three questions with one set of numerical simulations. The simulations are based on the GR-Astro code (formerly called GR-3D) constructed in the NASA Neutron Star Grand Challenge project [5] and the NSF Astrophysics Simulation Collaboratory (ASC) project [6]. For the construction of the code and the classes of validation tests we have carried out for it, see 7, 8, 9, 10]. The GR-Astro code solves the coupled set of the Einstein equations and the general relativistic hydrodynamic equations with a realistic EOS. It will be released to the community through the ASC portal [6] upon completion of the project.

In this paper, we report on simulations of NSs constructed with the Lattimer-Swesty 11] (LS) EOS, which has been used in various neutron star studies (all existing simulations based on the LS EOS that we are aware of are based on Newtonian gravity and hence cannot answer questions of collapse). To the best of our knowledge, our simulations represent the first set of general relativistic 3D simulations based on a realistic EOS (for polytropic EOS simulations, see 12]). In this study, we use neutron stars of rest (baryonic) mass 1.6 $M_{\odot}$ (corresponding to an $\mathrm{ADM}$ mass of $1.4 M_{\odot}$ in isolation), with a radius of $13.8 \mathrm{~km}$ (proper distance). The merged object has a rest mass of $3.2 M_{\odot}$ which is considerably lower than the critical mass of $3.67 M_{\odot}$ in the LS EOS with our choice of parameters. Nevertheless, we find that the merged object will promptly collapse to a black hole within a dynamical timescale. An apparent horizon is found engulfing the shock wave at $0.15 \mathrm{~ms}$ after the two stars have touched, with time measured at infinity, i.e. at the edge of the computational grid, which is $47 \mathrm{~km}$ away from the 
collision center. The prompt collapse is verified for both the head-on collision case and an off-axis collision (the axisymmetry is broken by an impact parameter of $1 / 2$ stellar radius). This study demonstrates that dynamical effects are strong enough to cause a prompt collapse even when the total mass is below the single-star critical mass, and that this result is not a consequence of exact axisymmetry. We hence post the following "Prompt Collapse Conjecture": For head-on and near head-on collisions of neutron stars described by a generic equation of state and infalling from rest at infinity, there exists a window in the rest mass of the merged object, below the critical single-star rest mass, where prompt collapse to a black hole can occur. The claim of the "near headon" part means that the prompt collapse is stable with respect to small perturbations of the initial velocity.

The Setup. In this paper we use our implementation of the LS EOS as described in 13. We use the LS EOS in a tabular form with rest mass density and specific energy density as the two independent thermodynamic variables, which are evolved using the general relativistic hydrodynamic (GR-Hydro) equations (the lepton to baryon ratio is set to a constant (0.1) in all simulations in this paper). As in the Newtonian LS EOS simulations of [14, 15], we set the initial specific energy density of the NSs at a relatively large value of $0.9 \mathrm{Mev}$. The ADM mass and rest mass as a function of central rest mass density for a single static NS is given in Fig. 1. We see that the critical rest mass of an LS EOS star is at 3.67 $M_{\odot}$. In our simulation we use NSs with a rest mass of 1.6 $M_{\odot}$ (marked by a $\mathrm{X}$ in Fig. 1$)$. The merged object is hence guaranteed to have a rest mass below the critical single-star mass. In Fig. 1 we have also plotted the corresponding curves for the case of a polytropic EOS with $\Gamma=2$ and $K=1.16 \times 10^{5} \mathrm{~cm}^{5} / \mathrm{g} \mathrm{s}^{2}$ for comparison.

In the head-on collision case, we put the two stars at a proper distance of $d=42 \mathrm{~km}$ apart (about $3 R$ separation, $R=$ radius of star) along the z-axis, and boost them toward one another at the speed (as measured at infinity) of $\sqrt{G M / d}$ (the Newtonian infall velocity). The metric and extrinsic curvature of the two boosted stars are obtained by (i) adding the off-diagonal components of the metric, (ii) adding the diagonal components of the metric and subtracting 1, (iii) adding the components of the extrinsic curvature. The resulting matter distribution, momentum distributions, conformal part of the metric, and transverse traceless part of the extrinsic curvature are used as input to York's procedure [16] for determining the initial data. The initial data satisfies the complete set of Hamiltonian and momentum constraints to high accuracy (terms in the constraints cancel to $10^{-6}$ ), and represents two NSs in head-on collision.

The initial data is then numerically evolved by solving the coupled Einstein GR-Hydro evolution equations with numerical methods described in [7]. The simulations reported here use the " $1+\log$ " slicing [17]. The simula-

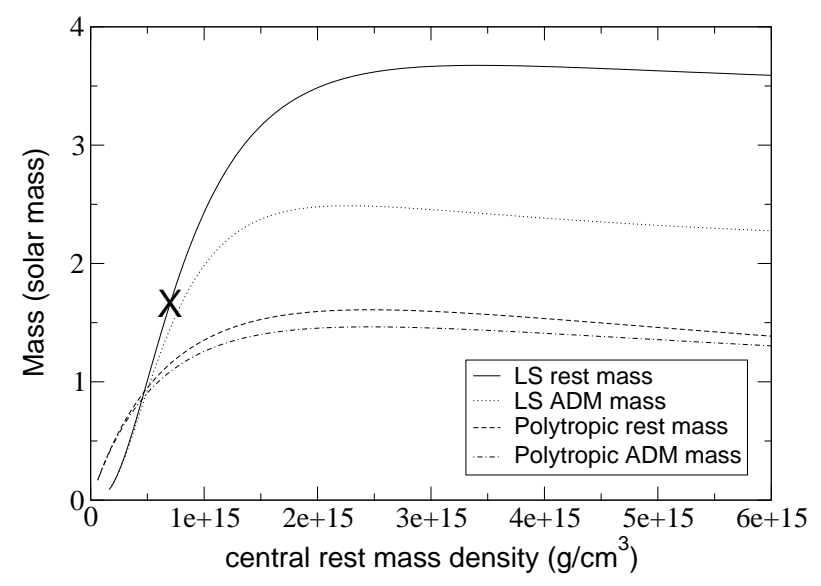

FIG. 1: The ADM mass and rest mass vs. central rest mass density of a static NS with the LS EOS. The corresponding curves for a polytropic EOS with $\Gamma=2, K=$ $1.16 \times 10^{5} \mathrm{~cm}^{5} / \mathrm{g} \mathrm{s}^{2}$ are plotted for comparison.

tions have been carried out with resolutions ranging from $\Delta x=0.74 \mathrm{~km}$ to $0.3 \mathrm{~km}$ (28 to 70 grid points across each NS) for convergence and accuracy analysis. We find that the constraint violations rise linearly throughout the evolution, and converge to zero with increased numerical resolution. The total rest mass of the system is conserved to better than $0.2 \%$ throughout the simulations.

The Results. In Fig. 2a, we show the collapse of the lapse along the $z$ axis from $t=0 \mathrm{~ms}$ to $t=0.37 \mathrm{~ms}$ at intervals of $0.0926 \mathrm{~ms}$. (With the reflection symmetry across the $z=0$ plane and the axisymmetry of the head-on collision, we only need to evolve the first octant.) By the time $t=0.37 \mathrm{~ms}$, the lapse has collapsed significantly. Fig. $2 \mathrm{~b}$ shows the corresponding evolution of the $z z$ component of the metric function. The "grid stretching" peak, characteristic of a black hole evolved in a singularity avoiding slicing, is apparent.

Fig. 3 shows contour lines in the $y=0$ plane of the $\log$ of the gradient of the rest mass density $\log \left(\sqrt{\nabla^{i}(\rho) \nabla_{i}(\rho)}\right)$ at time $t=0.37$ ms. Sharp changes in rest mass density (where contour lines bunch up) indicate shocks. We see that the shock front is at $7 \mathrm{~km}$ in the $\mathrm{x}$ direction, and $10 \mathrm{~km}$ along the $z$ direction (the collision axis) and has not yet reached the back end of star (at $14.5 \mathrm{~km})$. At this point the shock front is still moving outward in coordinate space, although it is completely engulfed by the apparent horizon, as seen in Fig. 4 below.

In Fig. 4, we show the intersection of the apparent horizon $(\mathrm{AH})$ with the $x-z$ plane. To confirm the location of the $\mathrm{AH}$, convergence tests, both in terms of resolution and in terms of location of the computational bound- 

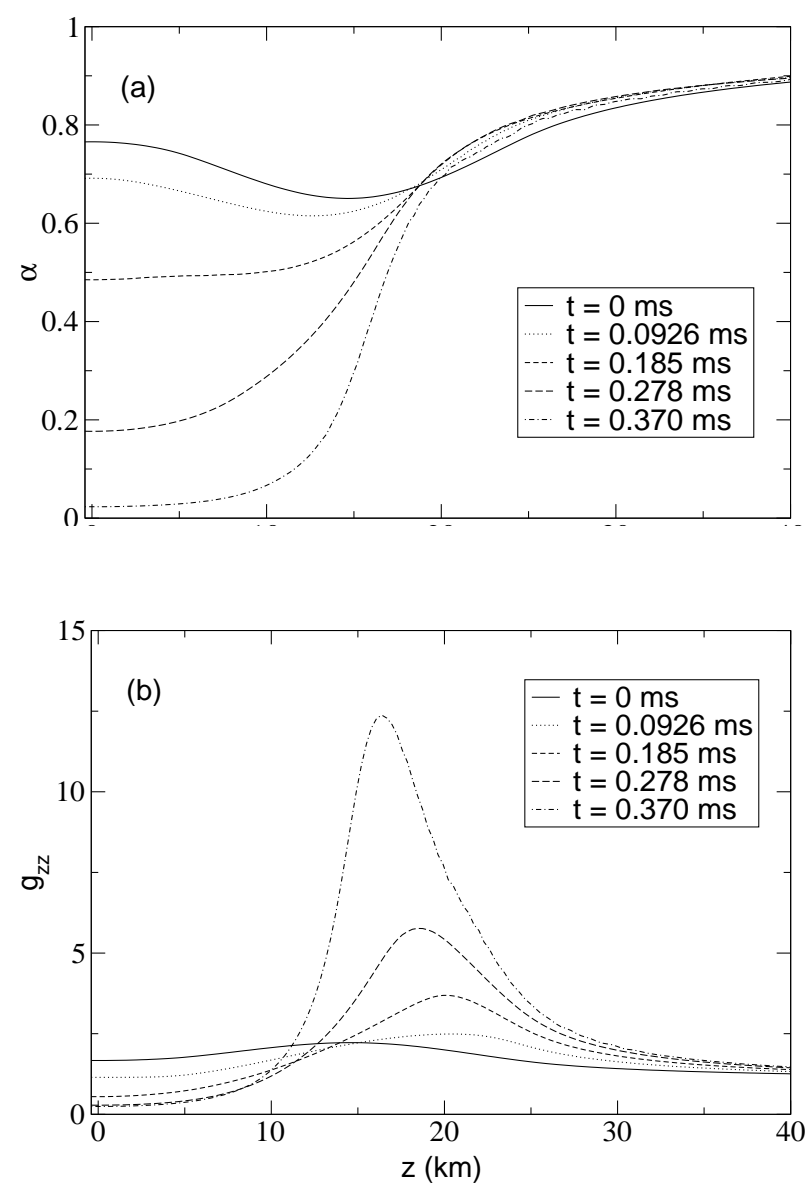

FIG. 2: The lapse $\alpha$ and metric component $g_{z z}$ is displayed along the $z$-axis at various times for the head-on collision of 2 NSs using the LS EOS. This simulation used $163^{3}$ grid points, with $\Delta x=0.3 \mathrm{~km}$.

ary, have been carried out. The solid, short dashed, long dashed and dotted lines correspond to the $\mathrm{AH}$ locations at resolutions of $\Delta x=0.74,0.52,0.37$ and $0.30 \mathrm{~km}$. The inset is a close-up view near the $z$-axis. We see that the location of the $\mathrm{AH}$ converges in a first order manner. We emphasize the importance of demonstrating the convergence of the final results in a numerical simulation. Such a validation of the final result is computationally expensive but nevertheless indispensable, as a short term convergence test of the numerical code does not guarantee the convergence of the final result at late time. In Fig. 5 we show the hamiltonian constraint converging linearly with respect to resolution for the whole duration of the numerical evolution.

Analysis of the Results. The above results demonstrate the phenomenon of a "sub-critical mass collapse": a prompt collapse of the merged object, despite the fact that its total rest mass is below the critical rest mass of a single NS.

One might question whether this result depends sen-

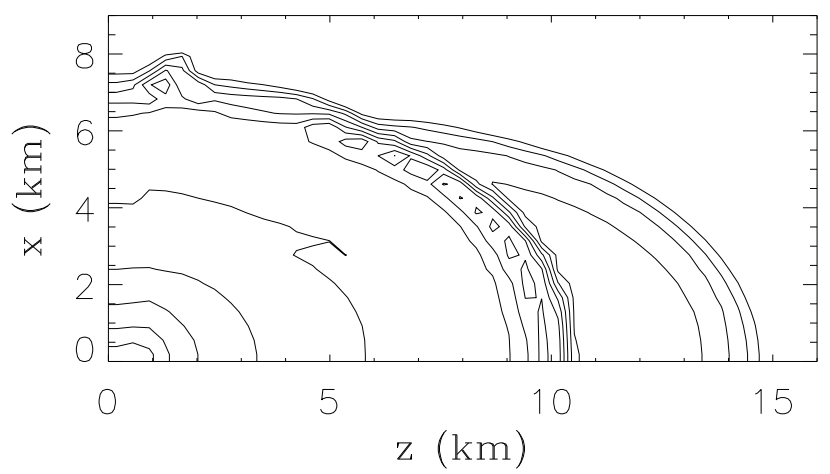

FIG. 3: Equally spaced contour lines of the log of the gradient of the rest mass density $\log \left(\sqrt{\nabla^{i}(\rho) \nabla_{i}(\rho)}\right)$, showing the shock front at $t=0.37 \mathrm{~ms}$.

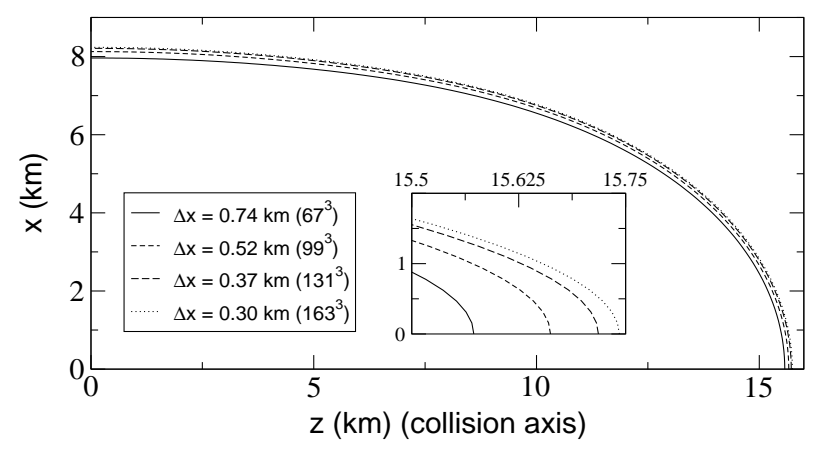

FIG. 4: The position of the $\mathrm{AH}$ in the $x-z$ plane at different resolutions, all at $t=0.37 \mathrm{~ms}$.

sitively on the choice of the initial velocity. As the star was only at about $3 R$ separation at the start of the simulation the infall velocity determined by the Newtonian formula is not accurate. We have verified that increasing or decreasing the initial velocity by $10 \%$ does not change the qualitative feature of the collision, and in particular the prompt collapse result. Likewise, our initial configuration while satisfying the constraints may not have exactly the same gravitational wave content and distortion of the NSs as one that represents free fall from infinity. We do not expect that to have a significant influence on the result of prompt collapse (although it will affect the gravitational waveforms). To verify this point we have also carried out simulations using slightly distorted stars and slightly modified extrinsic curvature for the construction of the initial data. We confirmed that the prompt collapse result is not affected.

One might also wonder whether this prompt collapse result is a consequence of the exact symmetry used. To determine this point we carried out simulations with the two initial NS moving in the $z$ direction but offset in the $x$ direction by half a radius (an impact parameter 


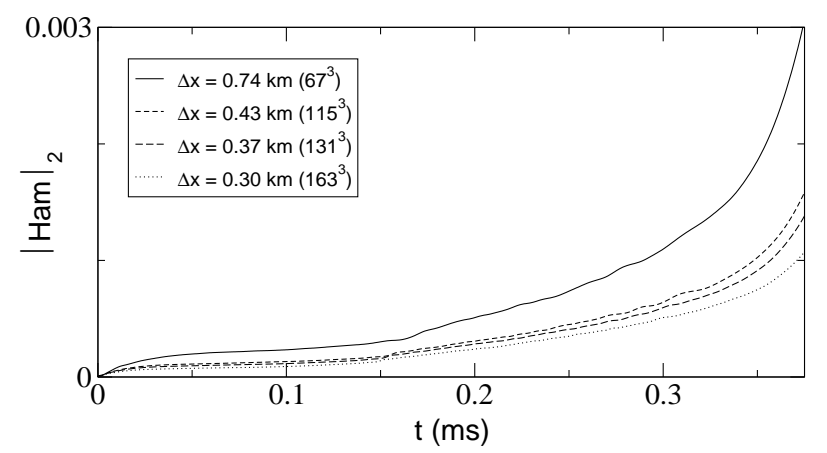

FIG. 5: The L2 norm of the hamiltonian constraint converging linearly with respect to resolution for the whole duration of the numerical evolution.

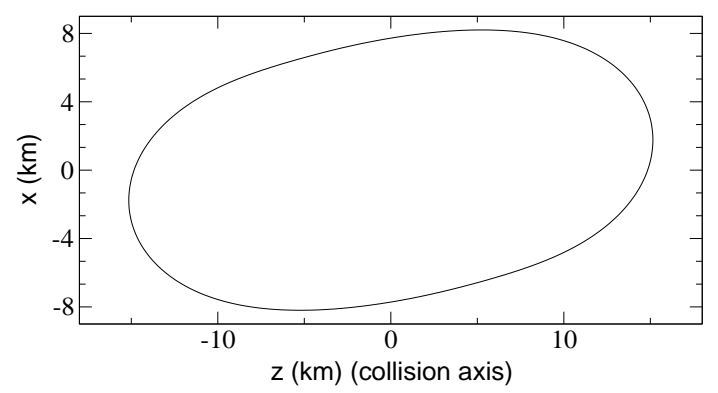

FIG. 6: The position of the AH at $t=0.37 \mathrm{~ms}$ for a near head-on collision using a spatial resolution of $\Delta x=0.37 \mathrm{~km}$.

of $6.9 \mathrm{~km}$ ). With this setup, the computation expense is significantly higher, as we can no longer evolve just an octant as in the axisymmetric case above. We found that the inclusion of a small impact parameter does not change the qualitative features of the collision, including the occurrence of prompt collapse. In Fig. 6, we show the apparent horizon found again at $t=0.37 \mathrm{~ms}$. We see that the $\mathrm{AH}$ is tilted with respect to the $z$ axis, but is very similar in shape and size to the head-on case.

In all the above cases, we have prompt collapses. We have confirmed that at a low enough mass, the merged object will not collapse but will instead merge, bounce, oscillate and form a stable NS. However, the determination of the dividing line between a final state black hole or a final state NS would require extremely high resolution whose computational expense is beyond what is available to our group.

Finally, one may also ask whether the sub-critical mass collapse occurs in the head-on collision of NSs described by a polytropic EOS. Preliminary studies with our 3D code show that, in fact, the dividing line in the rest mass of the final merged object between prompt collapse and non-prompt collapse is quite close to the critical rest mass of a single NS for the $\Gamma=2$ polytrope. We are currently working on an axisymmetric version of the code which we will use to not only explore this question, but to also explore the possible existence of type- 1 critical phenomena at the interface dividing the prompt collapse and non-prompt collapse cases.

Conclusions. In this paper we report on a subcritical mass collapse phenomenon in the head-on/near head-on collisions of NSs with a realistic EOS. We propose a "Prompt Collapse Conjecture": For head-on and near-head-on collisions of neutron stars described by a generic equation of state and infalling from rest at infinity, there exists a window in the rest mass of the merged object, below the critical single-star rest mass, where prompt collapse to a black hole can occur. This is the opposite of the Shapiro conjecture 1], which predicted no prompt collapse for all NS masses, including those above the single-star critical mass.

It has been argued in 18 that head-on/near head-on collisions of NSs could have a significant event rate, and could be a candidate for a sub-class of short gamma-ray bursts. The results of prompt collapse reported in this paper could have implications on the observation of such processes, with the prompt formation of the horizon cutting off the causal connection of the shock heated matter from outside observers. Note that we are not claiming a prompt collapse in inspiral coalescence of NSs.

Acknowledgements. We thank Luc Blanchet, K. Thorne, and C. Will for useful discussions, and LapMing Lin for comments on the manuscript. The simulations in this paper have made use of code components developed by several authors: BAM (multigrid solver) by B. Brügman; AH-FINDER (apparent horizon finder) by M. Alcubierre; CONF-ADM (evolution of the Einstein field equations), MAHC (evolution for the GRHydro equations), and IVP (conformal constraint solver) by M. Miller; PRIM-SOL (solver for the hydrodynamical primitive variables) by P. Gressman, ELS (LS-EOS tabular treatment) by E. Evans, and the CACTUS Computational Toolkit by T. Goodale et al.

Support for this research has been provided by the NSF KDI Astrophysics Simulation Collaboratory (ASC) project (Phy 99-79985), NASA Neutron Star Grand Challenge Project(NCCS-153), the NSF NRAC Project Computational General Relativistic Astrophysics (93S025), and the NASA AMES NAS.

[1] S. Shapiro, Phys. Rev. D 58, 103002 (1998).

[2] M. Miller, W.-M. Suen and M. Tobias, Phys. Rev. D 63, 121501(R) (2001)

[3] M. Miller, W.-M. Suen and M. Tobias, gr-qc/9910022 (1999)

[4] S. Shapiro, gr-qc/9909059 (1999) 
[5] http://wugrav.wustl.edu/Relativ/nsgc.html

[6] http://wugrav.wustl.edu/ASC/

[7] J. A. Font, M. Miller, W. -M. Suen and M. Tobias, Phys. Rev. D 61, 044011 (2000)

[8] J. A. Font, M. Miller, W. -M. Suen and M. Tobias, Phys. Rev. D Repository, EPAPS: E-PRVDAQ-61029004 (2000)

[9] J. A. Font, T. Goodale, S. Iyer, M. Miller, L. Rezzolla, E. Seidel, N. Stergioulas, W-M. Suen and M. Tobias, Phys. Rev. D 65, 084024 (2002)

[10] W.-M. Suen, Prog. Theor. Phys. Suppl. 136, 251 (1999)

[11] J. M. Lattimer and D. F. Swesty, Nucl. Phys. A, 535, 331 (1991)
[12] M. Shibata and K. Uryu, Phys. Rev. D, 61, 064001 (2000)

[13] http://wugrav.wustl.edu/Codes/GR3D/GR3D_EOS.html

[14] M. Ruffert, H.-Th. Janka and G. Schäfer, Astron. Astrophys. 311, 532 (1996)

[15] M. Ruffert, H.-Th. Janka, K. Takahashi and G. Schäfer, Astron. Astrophys. 319, 122 (1997)

[16] J. York, in Sources of Gravitational Radiation, edited by L. Smarr (Cambridge University Press, Cambridge, 1979).

[17] P. Anninos, K. Camarda, J. Massó, E. Seidel, W.-M. Suen and J. Towns, Phys. Rev. D 52, 2059 (1995)

[18] J. I. Katz and L. M. Canel, Ap. J. 471, 915 (1996) 